\title{
Urachal Adenocarcinoma: About 3 cases
}

\author{
I Adrif , S Daoudi, N Filali, S Ouguellit, H Abbahssain, H Mrabti, H Errihani
}

\begin{abstract}
This is a retrospective study conducted at the national institute of oncology in rabat over a period of 10 years, between 2007 and 2017, 3 cases of urachal adenocarcinoma were collected during this period.

We analyzed the clinical, histological, therapeutic and evolutionary aspects of this unusual tumor.

They were a woman and 2 men, whose average age was 47 years. 2 of the 3 patients were smokers. The main tell-tale sign was gross hematuria. All patients received palliative chemotherapy and overall survival was $33.3 \%$ at 2 years.
\end{abstract}

Index Terms-Adenocarcinoma, Chemotherapy, Urachal.

\section{INTRODUCTION}

The urachal tumor is an extremely rare disease entity. It represents $0.01 \%$ of adult cancers. It is a very aggressive tumor with a rapid progression and a very poor prognosis.

We report the experience of the medical oncology service of the National Institute of Oncology in Rabat.

\section{MATERIEL AND METHODS}

This is a retrospective study conducted at the national institute of oncology in Rabat over a period of 10 years, between 2007 and 2017, 3 cases of urachal adenocarcinoma were collected during this period.

We analyzed the clinical, histological, therapeutic and evolutionary aspects of this unusual tumor.

\section{RESULTS}

They were a woman and 2 men, whose average age was 47 years with extremes between 30 and 58 years. 2 of the 3 patients were smokers. The main tell-tale sign was gross hematuria associated with signs of bladder irritation.

According to Sheldon's classification 1 case was stage IIIC while the other 2 cases were classified stage IVb with pulmonary and lymph node metastases for both.

All patients received palliative chemotherapy with FOLFOX as the first-line protocol for 2 cases and 5FU CDDP for 1 case. One patient on FOLFOX had stability after 8 cures followed by maintenance with LV5FU2 for 4 months before

ADRIF Imane, Department Of Medical Oncology, National Institute Of Oncology, Rabat, Morocco.

DAOUDI Sara, Department Of Medical Oncology, National Institute Of Oncology, Rabat, Morocco.

FILALI Nadia, Department Of Medical Oncology, National Institute Of Oncology, Rabat, Morocco. progressing and the other two showed disease progression during treatment.

All patients received a second-line chemotherapy; FOLFIRI, FOLFOX and CMV. The CMV patient died a week after his first cure and the other two cases progressed under treatment. Only one patient was able to receive third-line chemotherapy with Gem-CDDP, but only 2 cures could be administered due to the deterioration of the general condition. Overall survival was $33.3 \%$ at 2 years.

\section{DISCUSSION}

The uraque is an embryonic remnant of allantoic origin described as a tube connecting the bladder dome to the umbilicus forming from gestational day 28. The first description of an urachus tumer was reported by Hue and Jacquin in 1863 [1].

These tumors represent 0.17 to $0.34 \%$ of bladder cancers. It can occur at any age, with extremes of 6 to 87 years and seems to most often affect males around the age of 50 .

Histologically, it is most often mucosecreting or non-mucosecreting adenocarcinoma [1].

In order to be characterized as originating from the uraque, the tumor must meet the Mostofi criteria [2]:

- The site of the tumor at the level of the dome or anterior surface of the bladder;

- The extra-vesical development of the tumor;

- The absence of involvement of the bladder urothelium bordering the tumor with the absence of glandular or cystic cystitis;

- The presence of uraquian remnants in association with the neoplasm;

- The absence of a primary digestive adenocarcinoma.

The clinical symptomatology is dominated by hematuria, which is seen in 65 to $85 \%$ of cases. We can have a tender suprapubic mass, abdominal pain, voiding disorders, umbilical oozing, mucous urinary secretions (25\% of cases), highly suggestive of a mucosecreting adenocarcinoma but not specific to urachal, and a umbilical deformity [3]

On the radiological level, ultrasound is central to screening and often provides the first diagnosis of a heterogeneous echostructural mass of the bladder dome [4]. It specifies the size of the tumor, its location, as well as the pseudo-cystic nature of the supra-bladder tumor. Calcifications are quite frequent observed in 50 to $70 \%$ of cases. Helical computed tomography remains the basic examination for this pathology, it is divided into two parts: an intravesical caudal part and a supravesical part, often cystic, encapsulated, median extending towards the umbilicus under the rectus 
muscles [4]. According to Narumi et al. [4], the extension of urachal carcinoma along the Retzius space, antero-superiorly to the dome of the bladder, distinguishes it from advanced bladder carcinoma, the latter having mainly intra-bladder growth. Likewise, the characteristic midline position of uraqual carcinoma just above the bladder and directly posterior to the white line is an important factor for CT diagnosis.

Cystoscopy makes it possible to visualize the intravesical part of the tumor and to perform biopsies revealing the glandular nature of the lesion of the dome and specifying the absence of cystic or glandular cystitis in the perilesional mucosa [4].

The Sheldon classification allows us to classify urachal cancers into four stages $[4,5]$ :

- Stage I: no invasion beyond the uraque mucosa.

- Stage II: the invasion is confined to the uraque.

- Stage III: local extension:

A: in the bladder.

$\mathrm{B}$ : in the abdominal wall.

$\mathrm{C}:$ in the peritoneum.

D: in other viscera than the bladder.

- Stage IV: metastasis:

A: to regional lymph nodes.

B: remote.

The differential diagnosis arises with primary adenocarcinoma of the bladder or bladder metastasis from prostate, ovarian or colic cancer [4].

A good knowledge of the precise extent of the disease is justified by the fact that the standard treatment is partial surgery: it performs "en bloc" exeresis of the umbilicus, urachus, nearby peritoneum, posterior and lateral fascia, of the bladder dome which is associated with pelvic lymph node dissection. It is necessary to check the soundness of the margins [6]. Optimal surgery, in the absence of lymph node involvement, controls the disease in over $50 \%$ of cases in a series from the M.D. Anderson Cancer Center [6].

Patients with extensive lymph node, peritoneal, and metastatic involvement have very short survival. The proposed chemotherapies are those which are active in colorectal tumors: 5-fluoro-uracil, leucovorin, oxaliplatin, irinotecan, cisplatin [6].

\section{CONCLUSION}

The rarity and clinical latency of urachal tumors often results in late diagnosis and poor prognosis.

\section{REFERENCES}

[1] B. Lallemand and al. Adénocarcinome de l'ouraque Annales d'urologie 37 (2003) 30-32.

[2] Mostofi FK, Thomson RV, Dean AL. Mucous adenocarcinoma of the urinary bladder. Cancer. 1955.

[3] Descazeaud A. Pathologie de l'ouraque Urachal diseases. Ann urol. 2007.

[4] I. En-nafaa Adénocarcinome de l'ouraque: une cause rare d'hématurie. Pan African Medical Journal. 2013.

[5] V.Dadhania and al. Adenocarcinoma of the urinary bladder Am J Clin Exp Urol 2015

[6] H. Boyle, et al. Tumeurs rares de la vessie bullettin du cancer Volume 97 Supplément 3 juin 2010. 